\title{
Optimization of a variable-stiffness skin for morphing high-lift devices
}

\author{
G A A Thuwis, M M Abdalla and Z Gürdal \\ Department of Aerospace Structures, Faculty of Aerospace Engineering, Delft University of \\ Technology, 2629HS Delft, The Netherlands \\ E-mail: G.A.A.Thuwis@tudelft.nl
}

Received 30 June 2010, in final form 20 September 2010

Published 11 November 2010

Online at stacks.iop.org/SMS/19/124010

\begin{abstract}
One of the possibilities for the next generation of smart high-lift devices is to use a seamless morphing structure. A passive composite variable-stiffness skin as a solution to the dilemma of designing the structure to have high enough stiffness to withstand aerodynamic loading and low stiffness to enable morphing is proposed. The variable-stiffness skin is achieved by allowing for a spatial fibre angle and skin thickness variation on a morphing high-lift system. The stiffness distribution is tailored to influence the deformation of the structure beneficially. To design a realistic stiffness distribution, it is important to take aerodynamic and actuation loads into account during the optimization. A two-dimensional aero-servo-elastic framework is created for this purpose. Skin optimization is performed using a gradient-based optimizer, where sensitivity information is found through application of the adjoint method. The implementation of the aero-servo-elastic environment is addressed and initial optimization results presented. The results indicate that a variable-stiffness skin increases the design space. Moreover, the importance of taking the change in aerodynamic loads due to morphing skin deformation into account during optimization is demonstrated.
\end{abstract}

\section{Introduction}

Aerospace engineers search constantly for new technologies and ways to improve existing technologies in an attempt to revolutionize or optimize air travel. An increasing awareness of environmental issues has increased the pressure on today's aeroplane manufacturers to find novel concepts to achieve increased fuel economy and better noise reduction in their products, whilst thinking about aircraft end-of-life issues such as recycling. A large branch of the aerospace research conducted worldwide is focused on morphing aeroplane structures. Morphing allows an aeroplane to change its shape depending on the mission requirements. It is believed this will lead to the enhancement of the versatility and efficiency of future aircraft.

The application of morphing structures in aeroplanes is not new and can be traced back to the first motorized flight by the Wright brothers [1]. During their historic flight in 1903, wing twist was used to control the Wright Flyer I. Since then, a wide range of morphing structures have been developed and tested. These morphing structures are commonly divided into three categories depending on the scale:
- large scale morphing related to changes in wing area, span and sweep;

- medium scale morphing with changes in camber, twist and aerofoil shape;

- small scale morphing to alter the local aerofoil.

Examples of all three categories of morphing wing structures can be found in the review papers on recent and past activities in morphing technology in aircraft by Sofla et al [2] and Rodriguez [3].

The focus of the present paper is medium scale morphing where changes in aerofoil shape and camber are the most important applications. Changing wing camber is currently widely applied by commercial aeroplanes through the use of high-lift devices to increase lift and enable low speed flight during take-off and landing. According to Wlezien et al [4] these high-lift devices account for half of the mass and cost of an aircraft wing due to their complexity. It would be a real achievement if these high-lift devices could be replaced by gapless, less complex morphing structures. Within the European project 'Smart High Lift Devices for Next Generation Wings' (SADE) [5] research is aimed at the 
development of morphing alternatives to replace conventional high-lift devices. The ultimate goal is to find a morphing alternative, or alternatives as more solutions might be plausible, to create significant drag and noise reductions for new aircraft.

The design and creation of a morphing mechanism is unfortunately not the only key to a successful implementation of a morphing high-lift system on an aircraft wing. The aerodynamic outer surface also needs to be defined using some type of skin. Designing a morphing skin poses a dilemma, as the skin is required to be flexible to allow for actuation, while at the same time it is required to have the necessary stiffness needed to diffuse the aerodynamic forces to the support structure. A wide range of solutions for morphing skins have been developed and most are described in a review paper by Thill et al [6]. Skins which allow for shape changes can be created through stiffness change or stiffness tailoring. Stiffness change can, for example, be achieved using shape memory polymers [7] which are variable-stiffness materials that have a structural (stiff) and morphing (flexible) mode which are typically triggered by changes in temperature. A corrugated composite sheet $[8,9]$ is an example of a stiffness tailored skin which has a reduced bending stiffness in one direction. Bending stiffness reduction can also be achieved through the use of a multilayered skin [10] consisting of multiple unbonded thin layers. In the present work, a skin concept is sought which allows for fast deployment of the morphing system while requiring no or as little energy, e.g. heat, electricity, as possible. A passive skin concept requiring no pretreatment, e.g. application of a temperature difference, of the skin is as such preferable. Composites were selected for this design process because they give a large design freedom and are a mature technology.

It is important to include both aerodynamic and actuation loads during skin optimization to create a realistic stiffness distribution for morphing high-lift devices. This is required since a flexible skin might show significant deformations due to aerodynamic loads and hence alter these aerodynamic loads substantially. Therefore, an aeroservo-elastic (ASE) framework was created, coupling a twodimensional aerodynamic and structural solver to enable skin design using existing actuation topologies. The present framework differs from that introduced by Maute and Reich [11] for the topology design of a morphing actuation system in a few points of technique and in focus. Maute and Reich apply a 3D finite volume method to approximate an Euler flow, while in the present work a 2D panel code is applied to simulate the low speed aerodynamics: this is sufficient for the low speed situation considered here. The focus is on the design of the skin rather than the actuation system. Moreover, based on the observation [11] of unrealistic skin stretching, the skin strain was constrained in the present work.

\section{The skin concept}

Whenever a novel morphing actuation or support structure for a morphing high-lift device is created, there is the need to find a skin which is able to withstand the motion of the underlying morphing structure to guarantee skin continuity. Depending on the type of deformation, a specific skin should be selected which suits the required deformation type. If, for example, large skin stretching is required by the underlying actuation system, a skin with a large strain capability is a valid option. In the present work, the leading edge skin of a wing is designed for an actuation system that will invoke skin bending.

The response of the structure to actuation and aerodynamic loads is strongly dependent on the skin stiffness distribution. Application of a variable-stiffness [12] skin allows for the necessary stiffness variation required to achieve a target shape. Regions with a reduced bending or axial stiffness can be created to influence the deformation of the structure beneficially either by reducing maximum applied strains or by improving the matching of the deformed shape to the target. The requisite stiffness variation can be obtained either by changing the skin thickness or the fibre angles. In the latter case, the lay-up of such a skin can be produced using the now ubiquitous tow placement technology [13].

The main advantage of a variable-stiffness skin is that a better trade-off between stiffness under aerodynamic loads and flexibility under actuation loads can be obtained. The reduced stiffness (for flexibility) can be localized at specific regions that require a stiffness reduction to enable actuation. Compared to a constant-stiffness wing skin design, where the stiffness would be the same everywhere, a variable-stiffness skin facilitates increased stiffness in resisting aerodynamic loads. Smooth deformations, i.e. no abrupt sectional changes, are possible by applying a stiffness-tailored composite skin since the stiffness can be designed to facilitate such smoothness.

In the present work the scope of the thin composite skin concept by DLR [14] is extended by embedding the design procedure in an aero-servo-elastic framework and by extending the design space. The original DLR skin achieves variable stiffness through a thickness variation while in the present work the variable stiffness is created by spatially varying both the thickness and the fibre angle.

\section{Aero-servo-elastic framework}

It is important to include aerodynamic and actuation loads during the optimization of the skin stiffness distribution for application on morphing high-lift devices. This is required because a more flexible skin shows significant deformations due to the aerodynamic loads, thereby altering the aerofoil shape and hence the aerodynamic load of the wing. The aerodynamic load can be used advantageously to satisfy the optimization objective by including the aerodynamic influence from an early stage in the design process. An aero-servoelastic (ASE) framework was created for this purpose and this is described below.

\subsection{Aeroelastic analysis}

The ASE framework couples a two-dimensional nonlinear Euler-Bernoulli beam co-rotational element [15] to a twodimensional inviscid panel code [16]. The structural and aerodynamic model have a different mesh, so radial basis 
function [17] interpolation using Wendland's $C^{2}$ function [18] was applied to interpolate the forces and displacements between both meshes. The following set of nonlinear equations was solved to evaluate the static aeroelastic [19] deformation of the structure:

$$
\mathbf{f}_{\text {in }}(\mathbf{x}, \mathbf{u})=\mathbf{f}_{\mathrm{ext}}(\mathbf{x}, \mathbf{u}),
$$

where $\mathbf{x}$ is a vector containing the design variables, $\mathbf{u}$ is the unknown displacement vector, $\mathbf{f}_{\text {in }}$ is the internal force vector and $\mathbf{f}_{\text {ext }}$ is the external force vector, defined as

$$
\mathbf{f}_{\mathrm{ext}}(\mathbf{x}, \mathbf{u})=\mathbf{f}_{\mathrm{act}}(\mathbf{x})+\mathbf{f}_{\mathrm{aero}}(\mathbf{u}),
$$

with $\mathbf{f}_{\text {act }}$ the actuation force vector and $\mathbf{f}_{\text {aero }}$ the aerodynamic load vector. Equation (1) is solved using the normal flow algorithm [20] to trace the nonlinear equilibrium path. The linearized equilibrium equations used for the path following procedure take the form

$$
\left[\mathbf{K}(\mathbf{x}, \mathbf{u})-\mathbf{K}_{\mathrm{a}}(\mathbf{u})\right] \Delta \mathbf{u}(\mathbf{x})=\mathbf{f}_{\mathrm{ext}}(\mathbf{x}, \mathbf{u})-\mathbf{f}_{\mathrm{in}}(\mathbf{x}, \mathbf{u}),
$$

where $\mathbf{K}$ is the structural stiffness matrix, $\mathbf{K}_{\mathrm{a}}$ is the aerodynamic stiffness matrix and $\Delta \mathbf{u}$ is the unknown displacement increment vector. The aerodynamic stiffness matrix is obtained analytically by differentiating the panel code.

\subsection{Design variables}

The skin stiffness for an Euler-Bernoulli beam model is defined in terms of the axial stiffness $E A$ and the bending stiffness $E I$ of a beam segment, where $E$ is the modulus of elasticity, $A$ is the cross-sectional area of the element and $I$ is the second moment of inertia. The axial stiffness $E A$ and bending stiffness $E I$ for symmetric laminates are related to the ABD matrix as [21]

$$
E A=\frac{1}{\left[A^{-1}\right]_{11}}, \quad E I=\frac{1}{\left[D^{-1}\right]_{11}} .
$$

In the present work, the axial and bending stiffness of the skin beam elements is defined as

$$
E A=\beta E_{1} h, \quad E I=\frac{\alpha E_{1} h^{3}}{12}
$$

where $E_{1}$ is the modulus of elasticity of a composite material in fibre direction, $\alpha$ is a non-dimensional bending stiffness parameter, $\beta$ is a non-dimensional axial stiffness parameter and $h$ is the thickness of the skin. Ideally, the design of the composite skin would be based on the choice of a stacking sequence for each element. This poses problems when the thickness, i.e. number of layers of the laminate, is not defined. Moreover, it leads to optimization problems containing a large number of design variables. All the information about the stacking sequence of a composite skin is encapsulated in the parameters $\alpha$ and $\beta$. These will be directly used as design variables together with the thickness $h$. Note that axial stiffness $E A$ and bending stiffness $E I$ cannot be treated separately. The feasible region of the stiffness parameters, discussed in section 3.4, ensures the proper relation between $\alpha$ and $\beta$ for the design of a realistic skin.
While the actuation topology is assumed, in this study, to be predefined, the magnitudes of the actuation forces are also included as design variables. The actuation force vector $\mathbf{f}_{\text {act }}$ is defined as

$$
\mathbf{f}_{\mathrm{act}}=\mathbf{f} \cdot \mathbf{c},
$$

where $\mathbf{f}$ is a matrix where each vector contains the forces of an independent actuator and $\mathbf{c}$ is a scaling vector which defines the magnitude of the final actuation forces acting on the structure. The design variable vector is then composed as $\mathbf{x}=\{\boldsymbol{\alpha}, \boldsymbol{\beta}, \mathbf{h}, \mathbf{c}\}$.

\subsection{Optimization formulation}

The aero-servo-elastic framework makes it possible to optimize the skin stiffness distribution while simultaneously sizing the actuation loads. Ideally, direct performance measures, e.g. total drag, lift to drag ratio, etc, would be optimized subject to structural and aerodynamic constraints. Such an approach would require a much more refined aerodynamic analysis to be embedded in the design loop. This calls for a Navier-Stokes solver with some turbulence modelling such as Tau [22] for a high-lift design problem. To avoid the prohibitive associated computational costs, a target shape is predefined based on purely aerodynamic considerations. The task for the aero-servo-elastic framework is then simply to find an optimal stiffness distribution such that the predefined deformed shape is found when the structure is fully loaded by aerodynamic and actuation loads.

The target shape is defined in terms of a target curvature change $\kappa_{\mathrm{t}}$ between the deformed and undeformed configurations. The reason for matching curvature rather than deformation is that the pressure distribution in the wing leading edge region is quite sensitive to curvature. It is well known that using least-squares matching of the deformation may lead to oscillatory deformations with unphysical local curvatures [23]. Using this target curvature, the optimization objective function I is defined as

$\mathbf{I}(\mathbf{x}, \mathbf{u})=\int\left(\kappa-\kappa_{\mathrm{t}}\right)^{2} \mathrm{~d} s=\sum_{i=1}^{N}\left\{\frac{L_{i}}{2} \int_{-1}^{1}\left[\kappa_{i}(\eta)-\kappa_{t_{i}}(\eta)\right]^{2} \mathrm{~d} \eta\right\}$,

where $\kappa_{i}(\eta)$ and $\kappa_{\mathrm{t}_{\mathrm{i}}}(\eta)$ are the actual and target curvature distribution on panel $i$, respectively, $L_{i}$ is the length of panel $i$, $\eta$ is a non-dimensional length parameter along a panel and $N$ is the total number of panels.

The design variables for the skin stiffness cannot be chosen arbitrarily during the optimization if the goal is to find a feasible skin. The laminate thickness $h$ is bounded by a minimum thickness requirement for two reasons. Definition of a minimum thickness allows for a skin design that can take design regulations with respect to bird strike into account. Secondly, the final stiffness distribution of the optimized design has to be converted into a set of fibre angles. It is necessary to have at least two fibre angles to guarantee the ability to match the optimal skin stiffness with the stiffness defined through a set of fibre angles. In the present work symmetric, balanced laminates are used, so the minimum thickness $h$ is equal to eight layers. Moreover, the 
Table 1. AS4/8773 material properties [26].

\begin{tabular}{lllll}
\hline$E_{1}$ & $E_{2}$ & $G_{12}$ & $v$ & $h_{\text {ply }}$ \\
\hline $1.198 \times 10^{11} \mathrm{~N} \mathrm{~m}^{-2}$ & $9.08 \times 10^{9} \mathrm{~N} \mathrm{~m}^{-2}$ & $5.29 \times 10^{9} \mathrm{~N} \mathrm{~m}^{-2}$ & 0.32 & $1.83 \times 10^{-4} \mathrm{~m}$ \\
\hline
\end{tabular}

maximum allowable strain $\epsilon_{\max }$ is assumed to be equal to 4000 microstrain. The objective function as defined in equation (7) is thus subjected to

$$
\begin{array}{ll}
\frac{\epsilon_{i}}{\epsilon_{\max }}-1 \leqslant 0, & \text { for } i=1 \cdots N ; \\
8 h_{L}-h_{i} \leqslant 0, & \text { for } i=1 \cdots N ;
\end{array}
$$

where $h_{\mathrm{L}}$ is the thickness of a single lamina and $\epsilon_{i}$ is the total strain on panel $i$. The feasible region of the $\alpha$ and $\beta$ parameters is derived in section 3.4.

The gradient information required to perform the optimization is computed within the ASE framework using the adjoint method [24]. The sensitivity of the objective function I with respect to the design variables $\mathbf{x}$ is found using the following set of equations:

$$
\begin{gathered}
\boldsymbol{\psi}=-\left(\mathbf{K}-\mathbf{K}_{\mathrm{a}}\right)^{-T} \frac{\partial \mathbf{I}}{\partial \mathbf{u}}, \\
\frac{\mathrm{d} \mathbf{I}}{\mathrm{d} \mathbf{x}}=\frac{\partial \mathbf{I}}{\partial \mathbf{x}}+\boldsymbol{\psi}^{\mathrm{T}}\left(\frac{\partial \mathbf{f}_{\mathrm{in}}}{\partial \mathbf{x}}-\frac{\partial \mathbf{f}_{\mathrm{ext}}}{\partial \mathbf{x}}\right),
\end{gathered}
$$

where $\boldsymbol{\psi}$ is the adjoint variable vector. The optimization is performed using the gradient-based optimization procedure GCMMA by Svanberg [25].

\subsection{Feasible region of stiffness parameters}

The design variables $\alpha$ and $\beta$ encapsulate the information about the stacking sequence of the composite skin, and for this reason they cannot be arbitrarily chosen. The parameters $\alpha$ and $\beta$ will be constrained to the region generated by all possible stacking sequences. This region defines the feasible set of stiffness parameters.

The boundaries of several valid design spaces are depicted in figure 1. These design spaces are created by calculating the axial and bending stiffness matrices of a composite with $n$ layers using classical lamination theory. The values for $\alpha$ and $\beta$ can consequentially be calculated from the relations in equations (4) and (5). The upper right corner of the design space defines a laminate with all ply angles equal to zero, while the lower left corner defines a laminate with all ply angles equal to $90^{\circ}$. The corner points in between are found to consist of $0^{\circ} / 90^{\circ}$ cross-ply laminates. A quasi-isotropic skin is defined using $\alpha=\beta=.3901$.

The feasible design space for the stiffness parameters $\alpha$ and $\beta$ depends on the chosen material. In the present work the unidirectional pre-impregnated carbon fibre in an epoxy material AS4/8773 is used. The cured material properties of this material are given in table 1 . The twelve-layer laminate design space is chosen since it limits the number of constraints to six. This design space is conservative compared to the design spaces of thicker laminates. The set of constraints

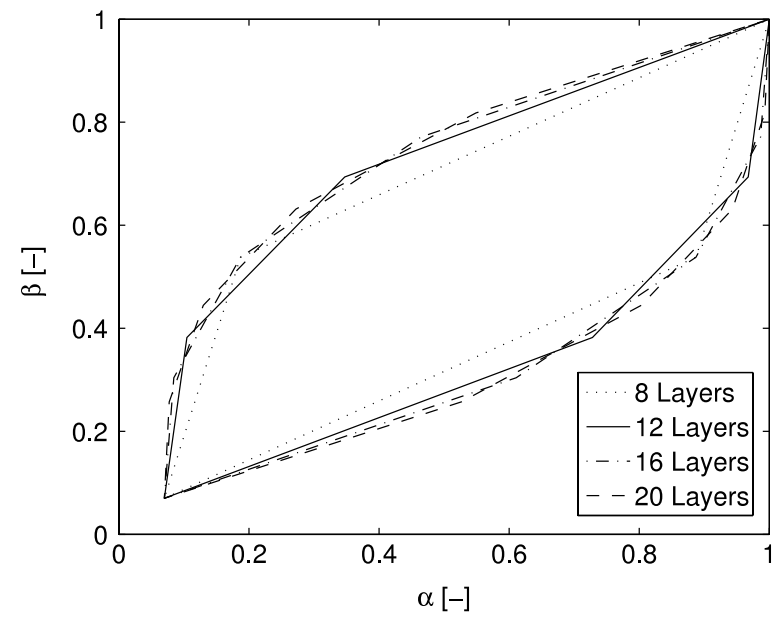

Figure 1. Design space for balanced symmetric laminates using AS4/8773.

in equations (8) and (9) are augmented with the following constraints representing the feasible region of laminates made of AS4/8773 material:

$$
\begin{array}{lc}
-\beta_{i}+0.4740 \alpha_{i}+0.0368 \leqslant 0, & \text { for } i=1 \cdots N \\
-\beta_{i}+1.3013 \alpha_{i}-0.5656 \leqslant 0, & \text { for } i=1 \cdots N \\
-\beta_{i}+9.4808 \alpha_{i}-8.4808 \leqslant 0, & \text { for } i=1 \cdots N \\
\beta_{i}-0.4694 \alpha_{i}-0.5306 \leqslant 0, & \text { for } i=1 \cdots N \\
\beta_{i}-1.2842 \alpha_{i}-0.2476 \leqslant 0, & \text { for } i=1 \cdots N \\
\beta_{i}-8.9981 \alpha_{i}+0.5595 \leqslant 0, & \text { for } i=1 \cdots N
\end{array}
$$

\section{Results}

A set of four optimization cases for a skin stiffness design on a morphing high-lift system were presented which neglect aerodynamic loads and two optimization cases with aerodynamic loading are considered. For all cases, the laminate was assumed to have a ply thickness of $1.83 \times 10^{-4} \mathrm{~m}$ and a minimum thickness of eight plies. The strain in the skin was limited to 4000 microstrain.

\subsection{Target shape, curvature and actuation system topology}

A representative next-generation wing (FNG) [27] was used within the SADE project. A deformed leading edge which allows the first $19 \%$ of the chord to deflect was optimized for landing manoeuvres and provided as input to the SADE project partners. The undeformed and deformed cross section of this wing are shown in figure 2 . The target curvature was thus predefined based on aerodynamic considerations. 


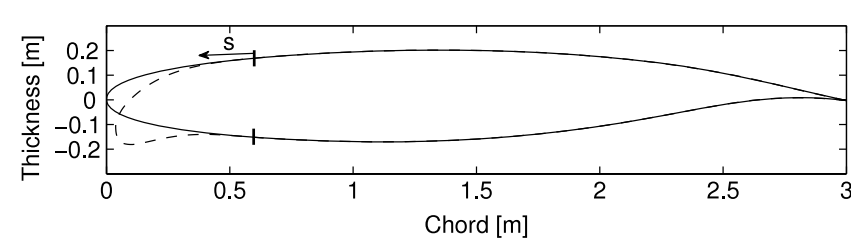

Figure 2. Undeformed (- - ) and target deformed (- - -) leading edge for FNG aerofoil.
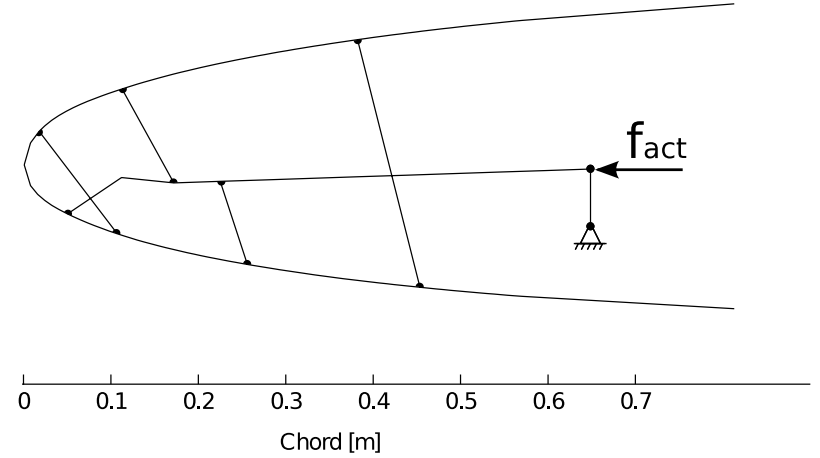

Figure 3. Actuation topology inside leading edge of FNG aerofoil.

Table 2. Nature of the skin stiffness variables for cases N1, N2, N3, $\mathrm{N} 4, \mathrm{~A} 1$ and $\mathrm{A} 2$.

\begin{tabular}{llll}
\hline & $\mathrm{N} 1$ and N2 & $\mathrm{N} 3$ and N4 & $\mathrm{A} 1$ and A2 \\
\hline Local variable & $\alpha, \beta$ & $\alpha, \beta, h$ & $\alpha, \beta, h$ \\
Global variable & $h$ & - & - \\
\hline
\end{tabular}

An initial, i.e. not optimized, actuation topology design by CIRA (Italian Aerospace Research Center) was used in the present work to show the capabilities of the ASE framework. This actuation system, depicted in figure 3, applies a set of rigid members and hinges to deform the skin through the application of a single actuator.

\subsection{Optimization results without aerodynamic load}

Four result cases are presented in this subsection to study the effect of fibre steering without accounting for aerodynamic loads. A single optimization took between 2 and $4 \mathrm{~h}$ on a standard PC (Intel Core 2 Duo E6750). A closer look is given to the effect of a variable-stiffness, variable-thickness skin on the ability to match a target curvature of a morphing high-lift system. The nature of skin stiffness variables for all cases is given in table 2, where a local variable indicates that the
Table 4. Result values for cases N1, N2, N3, N4, A1 and A2.

\begin{tabular}{lllllll}
\hline & $\mathrm{N} 1$ & $\mathrm{~N} 2$ & $\mathrm{~N} 3$ & $\mathrm{~N} 4$ & $\mathrm{~A} 1$ & $\mathrm{~A} 1$ \\
\hline$f_{\text {act }}(\mathrm{N})$ & 5961 & 6887 & 5963 & 7148 & 13688 & 14143 \\
$f_{\text {block }}(\mathrm{N})$ & - & - & - & - & 83930 & 84014 \\
$I(-)$ & 22.51 & 22.52 & 22.51 & 23.49 & 20.52 & 20.38 \\
\hline
\end{tabular}

variable is different on each element, and a global variable indicates that the variable is the same for all elements. The first two cases, denoted N1 and N2, as such optimize a constantthickness, variable lay-up skin. Cases N3 and N4, on the other hand, optimize a variable-thickness, variable-lay-up skin.

The initial values used to define the skin stiffness and actuation properties are tabulated in table 3. A quasi-isotropic lay-up was used as a starting point for cases N1 and N2 which differ in initial thickness. Cases N3 and N4 started with the same initial thickness, but differed in initial lay-up. The $\alpha$ and $\beta$ results of case N1 were used for case N3, while a quasi-isotropic lay-up was employed for case N4. The initial actuation load $f_{\text {act }}=1000 \mathrm{~N}$ was used for cases $\mathrm{N} 1-\mathrm{N} 4$. A total of 140 elements were used to discretize the leading edge skin on the structural model. No aerodynamic model of the aerofoil was required since the aerodynamic loading was neglected.

The goal of the optimization was to match the predefined target curvature of the leading edge skin. A comparison between the actual and target curvature for cases N1-N4 is depicted in figure 4 . It can be seen that the target curvature of the skin is not achieved by any of the four cases. The peaks in curvature are not matched because the strain limit is reached in these regions, as shown in figure 5. The deformed leading edges of cases N1-N4, shown in figures 6(a)-(d), reflect the difference between the actual and target shape. The actuation load required to deform the leading edge for cases N1-N4 is given together with the final objective function value in table 4 .

The difference in initial thickness between the constantthickness cases N1 and N2 does not result in a large difference in objective function value. The final thickness for both cases was equal to eight layers, as shown in figure 7. The axial and bending stiffness parameters, figures 8 and 9 , respectively, are nearly identical. The difference in initial skin thickness has, as such, no large effect on the final stiffness distribution.

The variable-thickness case N3 starts with the final stiffness values of constant-thickness case N1. Allowing the thickness to vary while using the final stiffness parameters $\alpha$ and $\beta$ of case $\mathrm{N} 1$ does not lead to an improvement in case N3 over N1. The skin thickness remains nearly constant, indicating that the results of case $\mathrm{N} 1$ cannot be improved by

Table 3. Input values for cases N1, N2, N3, N4, A1 and A2.

\begin{tabular}{llllccc}
\hline Case & $\alpha(-)$ & $\beta(-)$ & $h(\mathrm{~m})$ & $f_{\text {act }}(\mathrm{N})$ & $V_{\infty}\left(\mathrm{m} \mathrm{s}^{-1}\right)$ & AoA $(\mathrm{deg})$ \\
\hline N1 & 0.3901 & 0.3901 & $8 h_{\text {ply }}$ & 1000 & - & - \\
N2 & 0.3901 & 0.3901 & $12 h_{\text {ply }}$ & 1000 & - & - \\
N3 & Result N1 & Result N1 & $8 h_{\text {ply }}$ & 1000 & - & - \\
N4 & 0.3901 & 0.3901 & $8 h_{\text {ply }}$ & 1000 & - & - \\
A1 & 0.9 & 0.9 & $12 h_{\text {ply }}$ & 20000 & 90 & 6 \\
A2 & 0.95 & 0.95 & $11.5 h_{\text {ply }}$ & 20000 & 90 & 6 \\
\hline
\end{tabular}



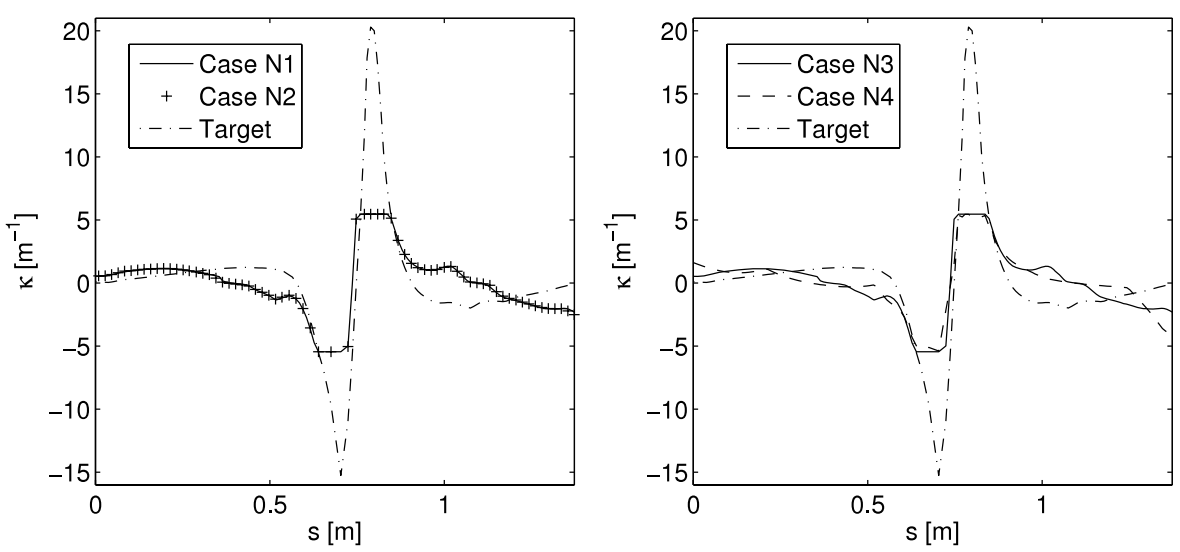

Figure 4. Actual versus target curvature for cases N1, N2, N3 and N4.
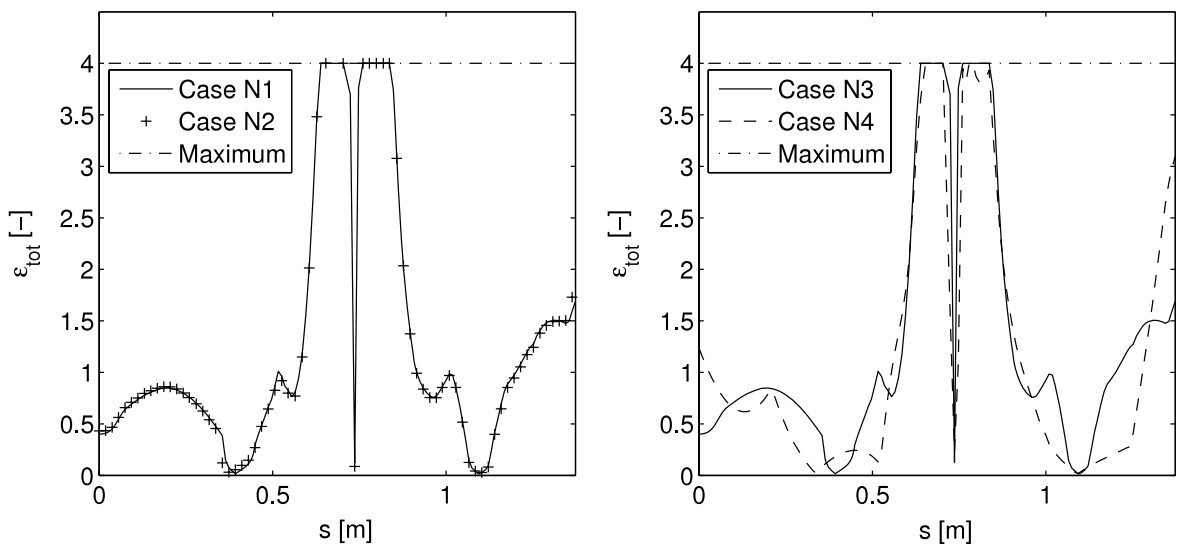

Figure 5. Total strain for cases N1, N2, N3 and N4.

an additional thickness variation. This makes sense since the optimization routine within the ASE framework wants to adjust the bending stiffness, which, according to equation (5), can be done by either tailoring $\alpha$ or $h$. The optimization routine within the ASE framework is forced to vary $\alpha$ in case N1 since the thickness should remain of equal magnitude over the entire skin. No further improvements are possible since the optimal bending stiffness $E I$ is already achieved if the skin thickness is allowed to vary on each element in case N3. The variation in axial stiffness parameter $\beta$ for cases $\mathrm{N} 1, \mathrm{~N} 2$ and $\mathrm{N} 3$ is required such that the combination of $\alpha$ and $\beta$ remains inside the feasibility domain defined in section 3.4.

The variable-thickness cases N3 and N4 start with a different initial distribution of stiffness parameters $\alpha$ and $\beta$. A larger difference of $\alpha$ and $\beta$ between cases N3 and N4 is shown in figures 8 and 9 , respectively, compared to cases N1 and N2. The final leading edge skin curvature in figure 4 is similar for cases N1, N2 and N3, but slightly different for case N4. A different combination of $\alpha$ and $h$ is used to define the final bending stiffness $E I$ of the skin for case N4 compared to cases $\mathrm{N} 1, \mathrm{~N} 2$ and $\mathrm{N} 3$. No large variation in axial stiffness parameter $\beta$ is required to remain within the feasible $\alpha, \beta$ domain for case N4 because $\alpha$ varies less compared to cases $\mathrm{N} 1, \mathrm{~N} 2$ and $\mathrm{N} 3$. The objective function value, shown in table 4, indicates that case N4 performs slightly worse than cases
N1-N3. However, the nature of the least-squares difference used to define the objective function together with the large objective function value indicates that it is likely that the optimization moved to a different local optimum for case N4.

\subsection{Optimization results with aerodynamic load}

Two optimization cases with aerodynamic loading, A1 and A2, are presented to highlight the capabilities of the ASE framework. Such an optimization including aerodynamic load needs 2-3 days to compute on a standard computer (Intel Core 2 Duo E6750). The 2D wing is simulated at an angle-of-attack of $6^{\circ}$ and an airspeed $V_{\infty}=90 \mathrm{~m} \mathrm{~s}^{-1}$. A blocked force $f_{\text {block }}$ is used to lock the actuation system in an undeformed state when full aerodynamic loading is applied. As such, the total force acting on the actuation system is the sum of the blocked force $f_{\text {block }}$ used to keep the actuation system in its undeformed state and the actuation force $f_{\text {act }}$ used to deflect the actuation system.

Due to the presence of the external aerodynamic load on the leading edge wing section, the skin requires an additional bending stiffness compared to cases N1-N4 where no aerodynamic load is simulated. As such, the initial values for the stiffness parameters $\alpha$ and $\beta$ are increased compared to cases N1-N4. The stiffness parameters $\alpha$ and $\beta$ are used to increase the stiffness rather than increasing the skin thickness. 


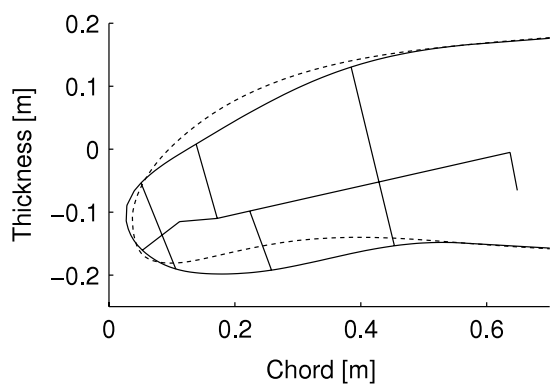

(a)

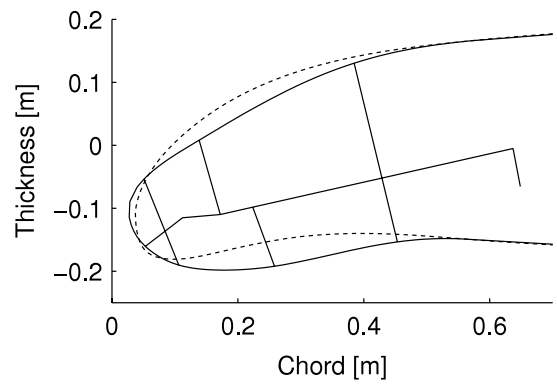

(c)

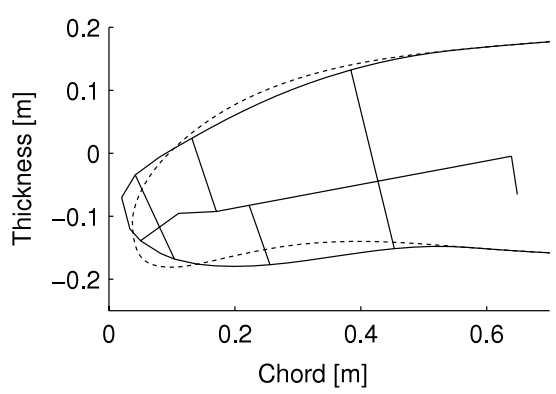

(e)

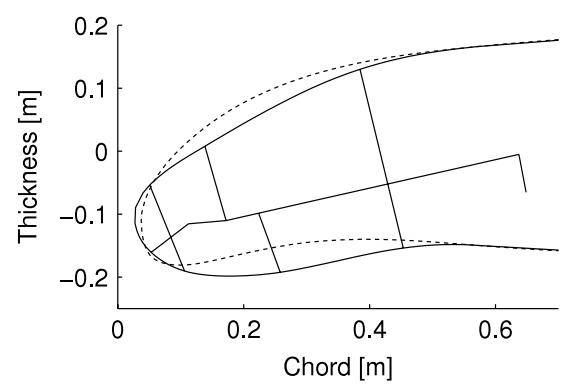

(b)

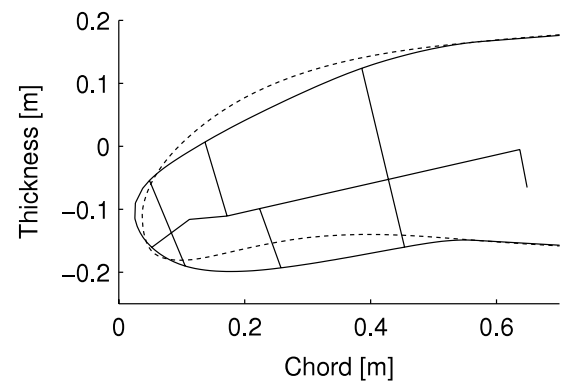

(d)

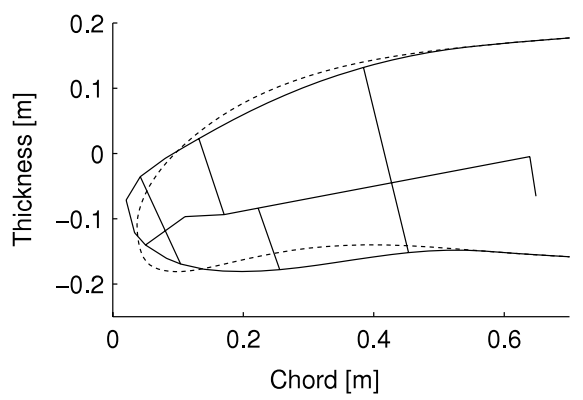

(f)

Figure 6. Deformed (_- ) leading edge versus target shape (-- -) without aerodynamic loading (cases N1-N4) and with aerodynamic loading (cases A1 and A2). (a) Case N1, (b) case N2, (c) case N3 and (d) case N4.
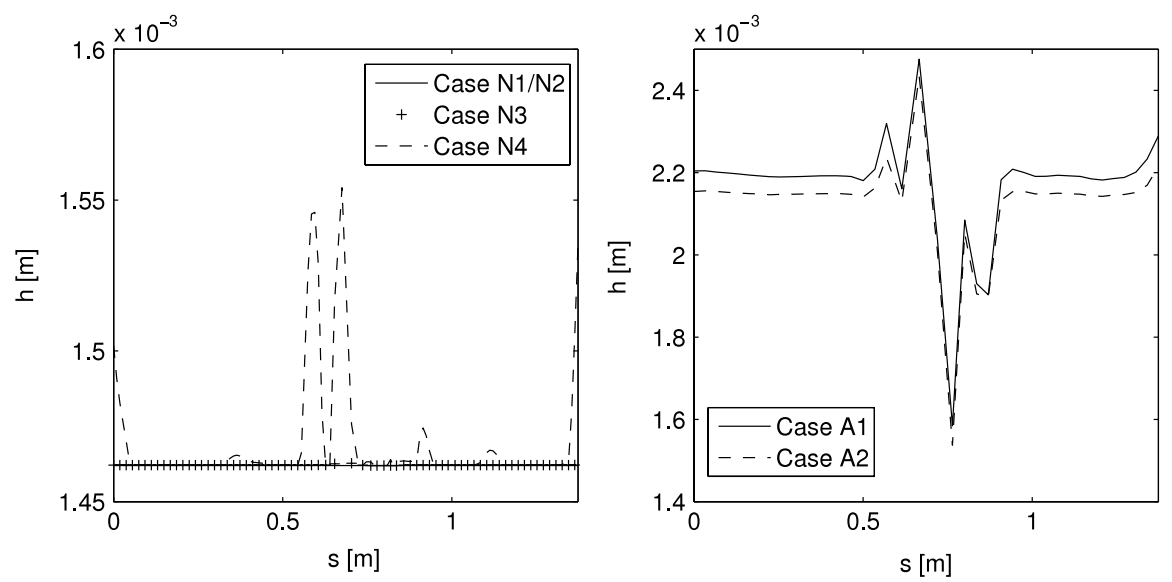

Figure 7. Thickness distribution $h$ for cases N1, N2, N3, N4, A1 and A2.

Increased skin thickness will have a negative effect on strain due to skin curvature. The initial stiffness values for the leading edge skin together with the initial actuation and aerodynamic properties are given in table 3 . The number of panels $N$ on the leading edge section of the structural model was equal to 40 . The number of panels was reduced to minimize the 

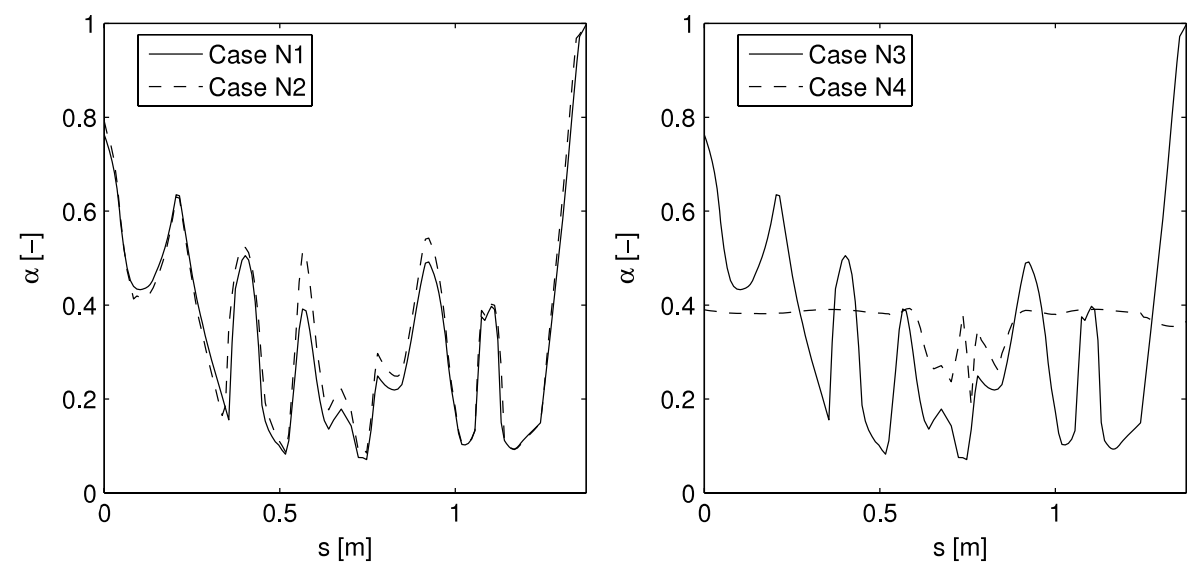

Figure 8. Bending stiffness parameter $\alpha$ for cases N1, N2, N3 and N4.
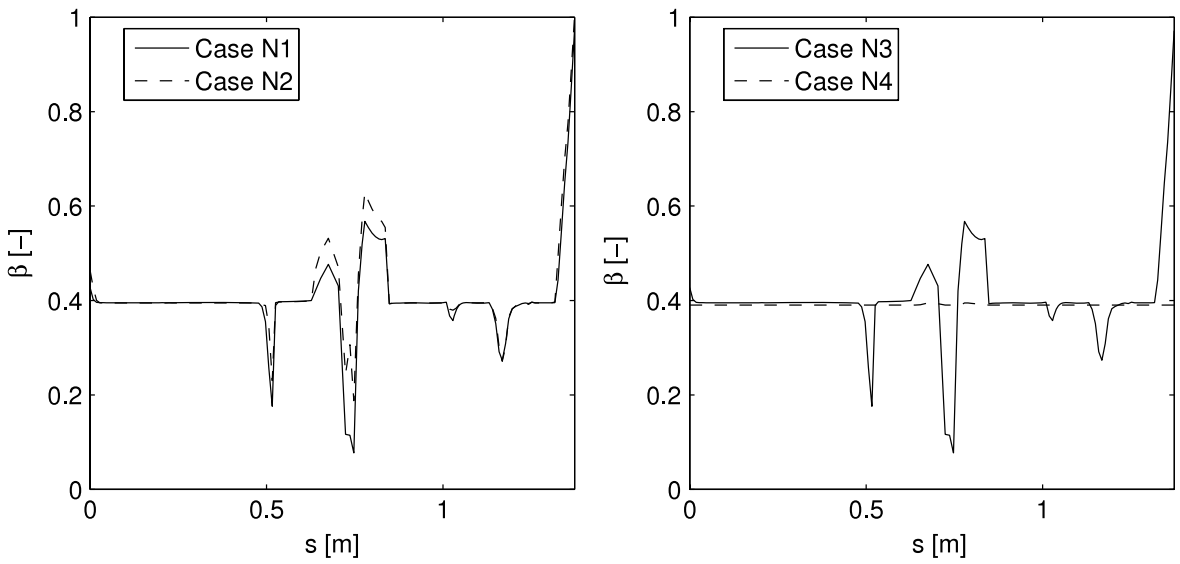

Figure 9. Axial stiffness parameter $\beta$ for cases N1, N2, N3 and N4.

computational effort required since the aerodynamic mesh is required to be finer than the structural mesh to get a good interpolation of forces and displacements between both meshes. The aerodynamic mesh therefore uses a total of 150 elements placed over the entire aerofoil using cosine spacing.

The final values for the objective function, blocked force and actuation force are given in table 4. The increase in actuation force for cases A1 and A2 compared to cases N1-N4, without aerodynamic load, is due to the aerodynamic loading applied to the morphing leading edge of a wing. The objective function value is slightly lower for cases A1 and A2 than the objective function of the cases without aerodynamics (N1-N4). This indicates that the curvature matches the target curvature to a slightly better extent as is confirmed in figure 10(a). The aerodynamic load acts beneficially to achieve the required target curvature: however, the final curvature is still relatively far from the target curvature. The reason for this inability to match the target curvature is twofold. One, the strain constraint is active in the leading edge region, as can be seen in figure 10(b) where the strain is plotted against the maximum allowable strain. Due to the higher skin thickness compared to cases N1-N4 without aerodynamic load, as shown in figure 7, the strain limit is reached sooner due to the dependence of the curvature strain on the skin thickness. Two, the second reason for the failure to converge to a matched solution is due to the actuation topology. The actuation system was not optimized to achieve the required deformation. As such, it forces the skin into a shape which deviates from the target shape which is related to the target curvature, as is shown in figures 6(e) and (f) for cases A1 and A2, respectively.

The stiffness parameters $\alpha$ and $\beta$ are plotted in figures 11(a) and (b), respectively, for a leading edge skin optimization including aerodynamic load. The axial stiffness parameter $\beta$ remains equal to the initial input value. Moreover, only a small change in bending stiffness parameter $\alpha$ can be seen near the nose region of the leading edge. The bending stiffness $E I$ is mostly altered through a thickness variation which is shown in figure 7 . The final values for the stiffness parameters $\alpha$ and $\beta$ are close to the stiffness parameter values of a $0^{\circ}$ laminate, i.e. $\alpha=\beta=1$. The fibres are thus almost aligned with the cross section of the wing and provide an additional bending stiffness required to withstand the external aerodynamic load acting on the skin. An increased bending stiffness can also be achieved by increasing the skin thickness, but this has a negative influence on the strain due to skin curvature.

The difference in initial condition for cases A1 and A2 remains visible in the results for $\alpha, \beta$ and $h$. The difference 


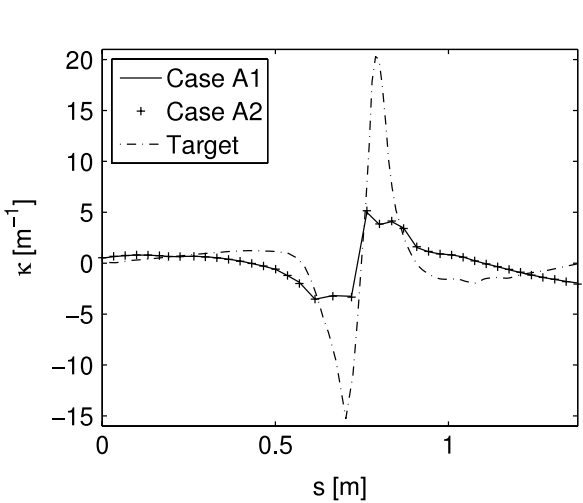

(a)

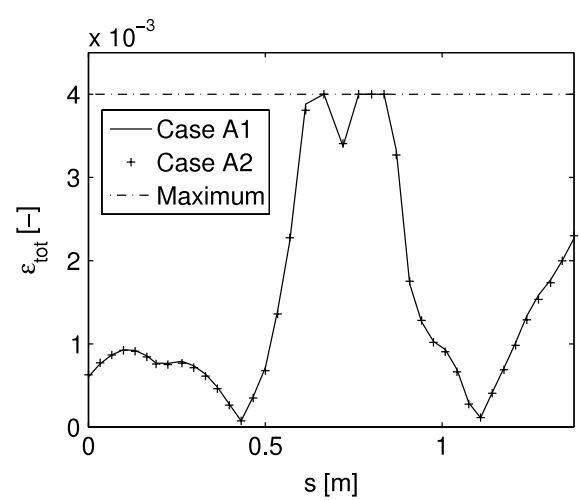

(b)

Figure 10. Curvature and strain results for cases A1 and A2 with aerodynamic load. (a) Actual versus target curvature. (b) Total strain.

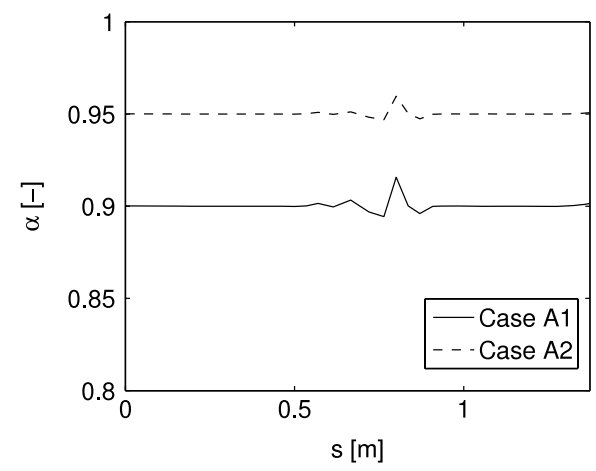

(a)

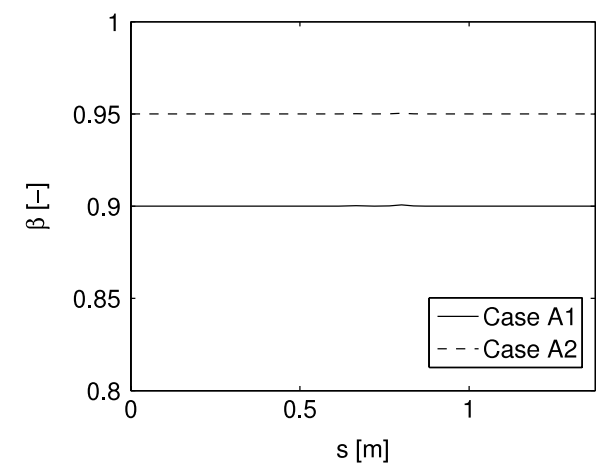

(b)

Figure 11. Optimized stiffness results for cases A1 and A2. (a) Bending stiffness parameter $\alpha$. (b) Axial stiffness parameter $\beta$.

in final stiffness variables has, however, no large effect on the final objective function value, target curvature and deflected shape. In the present work, the bending stiffness is the main parameter which is tailored during optimization since the skin is primarily loaded in bending. As such, tailoring of the axial stiffness through $\beta$ variation is not always necessary unless the combination of $\alpha$ and $\beta$ is outside the feasible domain for these parameters. The bending stiffness $E I$ can be changed by altering $\alpha$ and/or $h$. As such, a difference in initial values for $\alpha$ and $h$ can lead to different final values for these parameters, while the same bending stiffness $E I$ is achieved. Choosing good initial values for $\alpha$ and $h$ is important since a combination of high thickness and low value for $\alpha$ can result in the same bending stiffness as a combination of low thickness and a higher value for $\alpha$, but the higher thickness case will violate the skin strain constraint at a lower deflection. In that case a local optimum will be found which shows only a small deflection compared to the target shape. There is no guarantee that the global optimum is found since the problem is non-convex, but choosing different initial values for the stiffness variables helps in locating the best solutions.

\section{Conclusion}

A variable-stiffness skin for a leading edge of a wing is proposed for use on next-generation morphing high-lift devices. Variable stiffness is achieved by allowing for spatially varying fibre angles and thickness. An aero-servo-elastic framework for a seamless morphing leading edge high-lift system is presented to enable the design of such a skin in close interaction with the chosen actuation system and aerodynamic load. The first results neglect the aerodynamic load (cases N1$\mathrm{N} 4$ ), while the second set of results include the aerodynamic load (cases A1 and A2). Based on these results, the following conclusions can be formulated:

- a variable-stiffness skin has an increased design space compared to a constant-stiffness skin. From the results presented in this work, it becomes clear that, at certain regions on a morphing skin, a reduction or increase in stiffness is necessary to increase performance. Such stiffness changes cannot be achieved when only constantstiffness skins are considered.

- It is beneficial to have the actuation loads as part of the design variables, since this enables the design of a more coherent morphing high-lift system. The actuation loads can be minimized while the skin is optimized such that the combination skin/actuation gives the best performance with respect to the objective function.

- The aerodynamic loads have a large effect on the skin design and should therefore be included early in the design process. The presence of aerodynamic loads on the 
skin require a stiffer skin compared to the cases without aerodynamic loads.

The fact that none of the presented cases allow the target objective curvature to be achieved is mainly due to the fact that the actuation topology used in the present work was not optimized to facilitate the target curvature/shape. Future work will thus focus on the addition of an actuation topology optimization routine within the presented aero-servo-elastic framework. It is believed that a combined optimization of actuation topology and skin stiffness will enable the design of better performing and more coherent morphing high-lift devices. Further, inclusion of an improved aerodynamic solver with viscosity modelling will improve the simulation capability of high-lift flight. With such an improved aerodynamic solver, aerodynamic performance measures such as maximum lift can be used to define the objective function. The validity of results provided by the ASE framework will be checked against test data. Coupon tests will be performed which could, for example, involve the design and testing of a variable-stiffness plate with a predefined loading and target curvature. Such tests should evolve into the production and testing of a demonstrator model of a morphing high-lift system using a variable-stiffness skin. Finally, code optimization and use of high-performance computers should reduce the computational effort involved in the optimization of two-dimensional models with an increased number of degrees of freedom.

\section{Acknowledgments}

The authors would like to thank Professor K Svanberg for providing us with the Matlab code of his GCMMA optimizer. Furthermore we appreciate the input from Dr S Amaduri (CIRA) by providing us with an actuation topology to use in the present work. The financial support of the European Commission (FP7 grant no. ACP7-GA-2008-213442) has enabled the authors to pursue the design of variable-stiffness skins for application on morphing high-lift devices, support which is greatly appreciated.

\section{References}

[1] Wright O 1953 How We Invented the Airplane: An Illustrated History (New York: David McKay Company)

[2] Sofla A Y N, Meguid S A, Tan K T and Yeo W K 2010 Shape morphing of aircraft wing: status and challenges Mater. Des. 31 1284-92

[3] Rodriguez A R 2007 Morphing aircraft technology survey 45th AIAA Aerospace Sciences Meeting and Exhibit (Reno, Jan. 2007)

[4] Wlezien R W, Horner G C, McGowan A R, Padula S L, Scott M A, Silcox R J and Simpson J O 1988 The aircraft morphing program AIAA/ASME/ASCE/AHS/ASC Structures, Structural Dynamics, and Materials Conference and Exhibit (Long Beach, April 1998) AIAA-1998-1927

[5] SADE. Sade webpage www.smr.ch/sade/,2009

[6] Thill C, Etches J, Bond I, Potter K and Weaver P 2008 Morphing skins Aeronaut. J. 112 117-39

[7] McKnight H and Henry C 2005 Variable stiffness materials for reconfigurable surface applications Proc. SPIE—Int. Soc. Opt. Eng. 5761 119-26
[8] Yokozeki T, Takeda S I, Ogasawara T and Ishikawa T 2006 Mechanical properties of corrugated composites for candidate materials of flexible wing structures Composites A 37 1578-86

[9] Butler G 2007 Investigation of corrugated composite laminates for use in morphing wing applications Master's Thesis University of Bristol

[10] Ramrkahyani D S, Lesieutre G A, Frecker M and Bharti S 2005 Aircraft structural morphing using tendon-actuated compliant cellular trusses J. Aircr. 42 1615-21

[11] Maute K and Reich G W 2004 An aeroelastic topology optimization approach for adaptive wing design 45th AIAA/ASME/ASCE/AHS/ASC Structures, Structural Dynamics and Materials Conf. (Palm Springs, CA, April 2004) 2004-1805

[12] Gürdal Z and Almedo R 1993 In-plane response of laminates with spatially varying fiber orientations: variable stiffness concept AIAA J. 31 751-8

[13] Gürdal Z, Tatting B F and Wu K C 2005 Tow-placement technology and fabrication issues for laminated composite structures 46th AIAA/ASME/ASCE/AHS/ASC Structures, Structural Dynamics and Material Conf. (Texas, 2005) AIAA paper 2005-2017

[14] Monner H P, Kintscher M, Lorkowski T and Storm S 2009 Design of a smart droop nose as leading edge high lift system for transportation aircrafts 50th AIAA/ASME/ASCE/AHS/ASC Structures, Structural Dynamics, and Materials Conf. (Palm Springs, CA, May 2009) AIAA paper 2009-2128

[15] Crisfield M A and Moita G F 1996 A unified co-rotational framework for solids, shells and beams. Int. J. Solids Struct. 33 2969-92

[16] Katz J and Plotkin A 1991 Low-Speed Aerodynamics: From Wing Theory to Panel Methods (New York: McGraw-Hill)

[17] Beckert A 2000 Coupling fluid (CFD) and structural (FE) models using finite interpolation elements Aeros. Sci. Technol. 4 13-22

[18] Wendland H 1995 Piecewise polynomial, positive definite and compactly supported radial functions of minimal degree $A d v$. Comput. Math. 4 389-96

[19] Bisplinghoff R, Ashley H and Halfman R 1955 Aeroelasticity (Reading, MA: Addison-Wesley)

[20] Ragon S A, Gürdal Z and Watson L T 2002 A comparison of three algorithms for tracing nonlinear equilibrium paths of structural systems Int. J. Solids Struct. 39 689-98

[21] Gürdal Z, Haftka R T and Hajela P 1999 Design and Optimization of Laminated Composite Materials (New York: Wiley)

[22] Gerhold T, Friedrich O, Evans J and Galle M 1997 Calculation of complex three-dimensional configurations employing the DLR-tau-code AIAA Paper 97-0167

[23] Björck Å 1996 Numerical Methods for Least Squares Problems (Philadelphia, PA: SIAM)

[24] Maute K, Nikbay M and Farhat C 2003 Sensitivity analysis and design optimization of three-dimensional non-linear aeroelastic systems by the adjoint method Int. J. Numer. Methods Eng. 56 911-33

[25] Svanberg K 2002 A class of globally convergent optimization methods based on conservative convex separable approximations SIAM J. Optim. 12 555-73

[26] Blom A W, List J, Stickles P B and Gürdal Z 2008 Optimization of a composite cylinder under bending by tailoring stiffness properties in circumferential direction Int. Conf. on Engineering Optimization (Brazil)

[27] Dargel G, Hansen H, Wild J, Streit T, Rosemann H and Richter K 2002 Aerodynamische flügelauslegung mit multifunktionalen steuerflächen Deutscher Luft-und Raumfahrtkongress (Stuttgart, Sept. 2002) 